\title{
Improve the Robustness of Range-Free Localization Methods on Wireless Sensor Networks using Recursive Position Estimation Algorithm
}

\author{
Prima Kristalina $^{1,2}$, Wirawan ${ }^{1}$ \& Gamantyo Hendrantoro ${ }^{1}$ \\ ${ }^{1}$ Department of Electrical Engineering, Institut Teknologi Sepuluh Nopember (ITS) \\ Kampus ITS, Keputih Sukolilo Surabaya - Indonesia \\ ${ }^{2}$ Politeknik Elektronika Negeri Surabaya (PENS-ITS), Kampus ITS, \\ Keputih Sukolilo Surabaya - Indonesia \\ Email: prima@eepis-its.edu
}

\begin{abstract}
The position of a sensor node at wireless sensor networks determines the received data sensing accuracy. By the knowledge of sensor positioning, the location of target sensed can be estimated. Localization techniques used to find out the position of sensor node by considering the distance of this sensor from the vicinity reference nodes. Centroid Algorithm is a robust, simple and low cost localization technique without dependence on hardware requirement. We propose Recursive Position Estimation Algorithm to obtain the more accurate node positioning on range-free localization technique. The simulation result shows that this algorithm has the ability on increasing position accuracy up to $50 \%$. The trade off factor shows the smaller the number of reference nodes the higher the computational time required. The new method on the availability on sensor power controlled is proposed to optimize the estimated position.
\end{abstract}

Keywords: centroid; connectivity; localization; range-free; recursive.

\section{Introduction}

The position of a node in wireless sensor networks determines the performance of this network on the availability of data sensing. The accuracy of data sensing is influenced by the position of nodes sense the surrounding phenomena and the value of sensing data is depend on the distance between sensor node and its target.

Localization technique is essential in determining the position of random deployed sensors by a-priori knowledge of position. The position of unknown sensors is estimated from the vicinity reference sensors, which have knownposition based on the manually deployment or hardware utility such as GPS in measuring their geographic location. Actually, the reference sensors used as gateways for the other sensors in communicate with their server.

Received November $24^{\text {th }}, 2011$, Revised December $10^{\text {th }}, 2011$, Accepted for publication December $11^{\text {th }}, 2011$. Copyright (C) 2011 Published by LPPM ITB, ISSN: 1978-3086, DOI: 10.5614/itbj.ict.2011.5.3.4 
The two approach methods are intended in the localization technique based on the dependency on hardware utility, as reported by Liu, et al. [1] i.e range-based method and range-free method. Range-based method adopts ranging techniques and used inter node distance measurements to calculate the location of nodes. On contrary, the range-free method is cost-effective alternatives. Without direct distance ranging, the physical distance of a pair nodes is estimated by the proximity of them. The result of ranging-free method refers to the coarse estimated position.

Centroid is one of range-free localization method, as observed by Deng, et al. [2]. In this method, the position of the reference nodes surrounding an unknown node is averaged to obtain the estimated position of the respective node. Cheng, et al. [3] in the previous research described the shortcoming of Centroid Algorithm was the distribution model of reference nodes. If the nodes were deployed non-uniformly, their location errors were high enough. In Centroid Algorithm, the closer the reference nodes were placed to the unknown node, the greater the contribution of reference nodes dedicated.

In this paper we propose Recursive Position Algorithm to improve the coarse result obtained by Centroid Algorithm. The recursive technique used to increase the number of reference sensors derived from the settled node estimated before by centroid Algorithm. This technique has advantage in the usage of minimum reference node at initial process.

The rest of the paper is organized as follows. In section 2 the basic components of localization methods on wireless sensor networks is described. The similar effort on range-free localization research will be explained in section 3. The proposed Centroid Algorithms and Recursive Position Estimation technique imposed at Centroid Algorithms will be discussed at section 4. Finally, we conclude our works at section 5 .

\section{Localization System Components}

The localization system on wireless sensor networks is the effort to determine the geographic position of unknown nodes involved at the networks. The problem can be approximated by the network modeling, where the components are defined at this section.

\subsection{Problem Statement}

Wireless sensor networks contains $n$ nodes with $R$ communication range, distributed on two dimensional field $\mathrm{Q}=\left[\begin{array}{llll}0 & \mathrm{~s} & 0 & \mathrm{~s}\end{array}\right]$. In the simple approach, considered to use two symmetric communication links where any two nodes $u$ 
and $v, u$ received signal iff $v$ also received signal from $u$, with the same strength, $w$, as reported by Boukerche, et al. [4].

The network could be presented as a eucledian graph with the properties as follows:

$V=\left\{v_{1}, v_{2}, \ldots, v_{n}\right\}$ is the set of sensor node

$(i, j) \in E$ iff $v_{i}$ reaches $v_{j}$, and the distance between $v_{i}$ and $v_{j}$, is less than $R$.

$w(e) \leq R$ The weight of graph edge $e(i, j)$ i.e the distance between $v_{i}$ and $v_{j}$.

Some definition can be used to designate the state of the node.

Definition 1 (unknown nodes - $\boldsymbol{U}$ ). These are the sensor nodes in the network that their position is do not known. These nodes are called free nodes or dumb nodes. The aim of the localization system is to estimate the position of these nodes.

Definition 2 (settled nodes - S). These nodes are initially unknown nodes, where the estimated position is calculated by localization system. Later, these nodes together with reference nodes can be used for estimating the position of remain unknown nodes.

Definition 3 (reference nodes $-₹$ ). These nodes have initial position based on the deployment process on it, manually or by external means such as GPS. These nodes are called anchor or beacon.

Besides the definitions above, the localization system is defined as:

Definition 4 (localization problem): Given a multi hop network $G=(V, E)$ and a set of reference nodes $\boldsymbol{B}$, which have the position $\left(x_{b}, y_{b}\right)$ for all $b \in B$, and we will find the position $\left(x_{u}, y_{u}\right)$ as much as possible with $u \in U$ transforms the unknown node to be settled node $-\mathrm{S}$. 


\subsection{The Basic Components of Localization System}

De Oliveira, et al. [5] divided three basic components of localization system: distance ranging, position computation and localization algorithm, as shown at Figure 1. Each step of this component has influence to the performance of nodes estimated position accuracy.

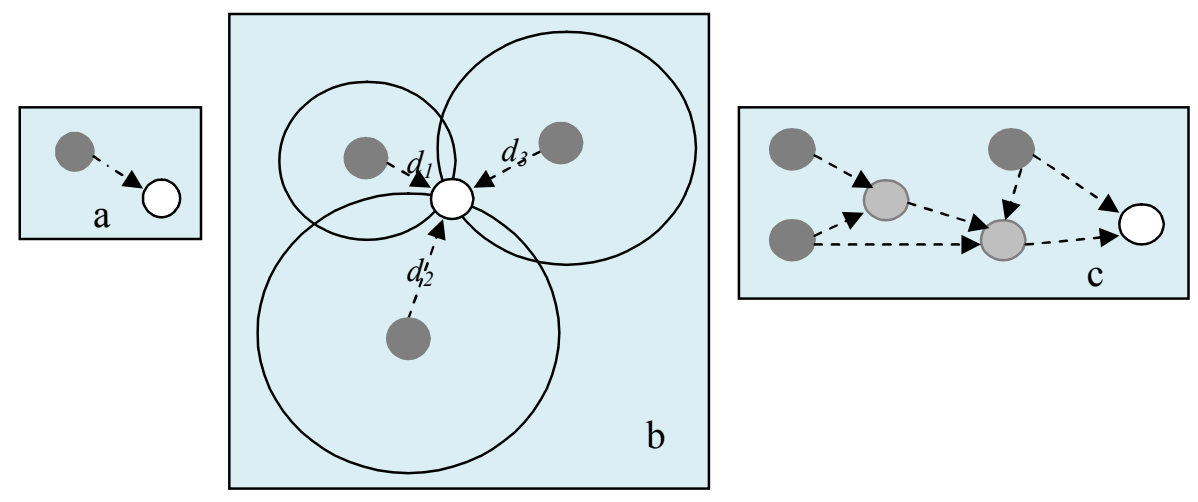

Figure 1 Three Basic Components of Localization System: a. Distance Ranging, b. Position Computation, c. Localization Algorithm.

\subsubsection{Distance Ranging}

The component of distance ranging, as described by Mao, et al. [6] has the responsibility on inter node sending information. It includes received signal strength (RSSI), time of arrival signal (TOA) and angle of arrival signal (AOA). The received information from this component will be processed by the other localization components. The resulting error of this component will affects to the accuracy of next process. Usually, the error occurs on this component due to the participation of noise measurement.

\subsubsection{Position Computation}

This component has the responsibility in calculating the position of sensor nodes based on distance information measurement obtained from reference nodes. The distance information will be calculated on this component yields estimated position of unknown nodes. Based on the requirement of distance ranging method, the computation process is divided into two categories: rangebased and range-free, as described by Langendoen, et al. [7]. Range-based method employs signal information received from the surrounding reference nodes then converts the information to the distance variable between reference nodes and the unknown nodes. The various methods of range-based measurement, as reported by Shi, et al. [8] are divided into Trilateral, 
Multilateral and Bounding Box. Range-free methods do not require the knowledge of distance ranging. It refers to the coarse position estimation resulting by the involved reference nodes. Centroid is the most popular algorithm on range-free method, as shown at Figure 2.

\subsubsection{Localization Algorithm}

This subject is the main component on localization system. The component determines how the information provided can be manipulated in such a way to obtain the estimated position of most or whole existing sensors. Several localization algorithms have been developed at two kinds of data processing system: centralized data processing and distributed data processing. The example of centralized algorithm is Multi Dimension Scale (MDS), whereas the algorithms implemented on distributed data processing are Ad-hoc Positioning System (APS) and Recursive Positioning Estimation (RPE).

\section{Related Works on Range-Free Position Computation}

The range-free computational method provides a promise challenge field observed by the researchers. The reason is the availability of it working without dependence on the hardware requirement. The estimated position error obtained by this method is higher relatively than the range-based method. Furthermore, this method has more beneficial in cost. Several works related with range-free position computation propose the algorithms to obtain the position accuracy precisely. The previous works on Centroid Algorithms and Recursive Position Estimation are listed below.

\subsection{Fundamental Centroid}

Bulusu, et al. [9] had proposed a proximity based algorithm. The algorithm was based on the proximity of adjacent nodes, i.e unknown nodes and its neighboring references. The coordinate of unknown nodes can be found at Eq. (1).

$$
\begin{aligned}
& \left(X_{\text {est }}, Y_{\text {est }}\right)=\left(\frac{x_{1}+x_{2}+\ldots+x_{N}}{N}, \frac{y_{1}+y_{2}+\ldots+y_{N}}{N}\right) \text { or } \\
& \left(X_{\text {est }}, Y_{\text {est }}\right)=\left(\frac{\sum_{i=1}^{N} x_{i}}{N}, \frac{\sum_{i=1}^{N} y_{i}}{N}\right)
\end{aligned}
$$


The result of the algorithm was a coarse approximation on node estimated position, as shown at Figure 2. This algorithm assumed that the distance weighted factor between all unknown and their reference nodes was similar.

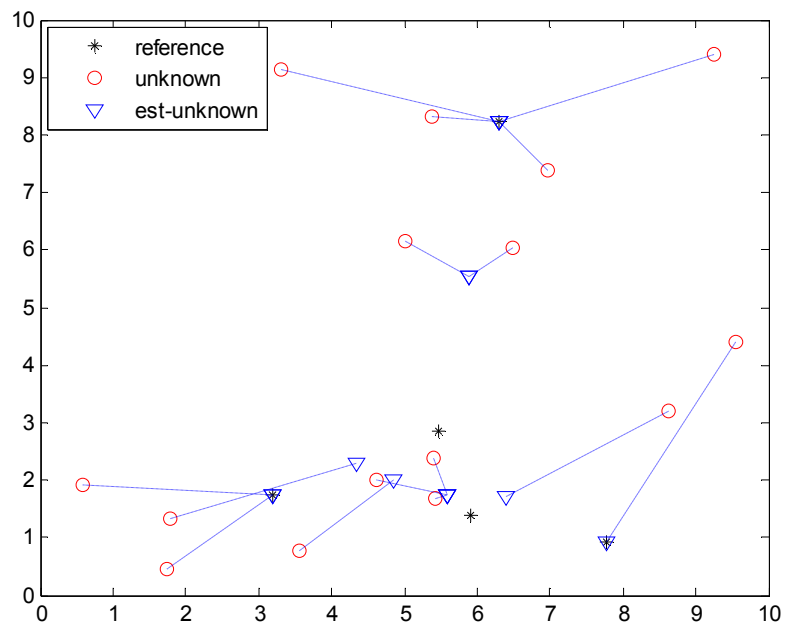

Figure 2 Fundamental Centroid for 5 Reference and 15 Unknown nodes.

\subsection{Centroid with TSK Fuzzy Weighted}

The weighted factor based on fuzification technique had been proposed by Yun, et al. [10]. In this paper the weighted factor based on fuzzy TSK (TakagiSugeno-Kang) had rule-model as below. This rule was used as the distance weight between reference and unknown nodes.

$$
\begin{aligned}
& \text { IF } x_{1} \text { is } C_{l}^{l} \text { and ... and } x_{N} \text { is } C_{N}^{l} \\
& \text { THEN } y^{l}=f_{l}\left(x_{1}, x_{2}, \ldots, x_{N} ; C^{l}\right)=c_{0}^{l}+c_{1}^{l} x_{1}+\ldots+c_{N}^{l} x_{N}
\end{aligned}
$$

For all $i=1, . ., M$, where $M$ was the number of rule, $C_{i}^{l}$ was a fuzzy set, then the average weight of $y^{l}$ and the estimated position coordinate of an unknown node was expressed at Eqs. (2) and (3), respectively.

$$
\bar{y}=\frac{\sum_{l=1}^{M} y^{l} w^{l}}{\sum_{l=1}^{M} w^{l}}=\frac{\sum_{l=1}^{M}\left(c_{0}^{l}+c_{1}^{l} x_{1}+\ldots+c_{N}^{l} x_{N}\right) w^{l}}{\sum_{l=1}^{M} w^{l}}
$$




$$
\left(X_{\text {est }}, Y_{\text {est }}\right)=\left(\frac{\bar{y}_{1} x_{1}+\bar{y}_{2} x_{2}+\ldots+\bar{y}_{N} x_{N}}{\sum_{i=1}^{N} \bar{y}_{i}}, \frac{\bar{y}_{1} y_{1}+\bar{y}_{2} y_{2}+\ldots+\bar{y}_{N} y_{N}}{\sum_{i=1}^{N} \bar{y}_{i}}\right)
$$

Using the fuzzy weighted, the estimated position resulting by Centroid algorithm had smoother and more accurate than the previous one.

\subsection{Centroid with Selected Anchor (SA Centroid)}

Deng, et al. [2] described the asymmetrical reference nodes distributed around the unknown node in a certain coverage area. This condition caused a large error on estimating the position of unknown node. To reduce the impact of asymmetrical problem, they used Triangle Centroid, e.g selected the three of surrounded reference nodes, then calculated the average of temporary coordinates. The process was repeated for the other subsets with the combination of different reference nodes, resulted another temporary coordinates. The final estimated coordinate for each unknown was the average of its temporary coordinates.

Let $A^{i}=\left\{X_{1}^{i}, X_{2}^{i}, \ldots, X_{l}^{i}\right\}$ be the set of $N$ reference nodes around $i$ unknown nodes, with $X_{R P}^{i}=\left(X_{R P}^{i}, Y_{R P}^{i}\right)$ was the coordinate of the nearest reference node. Then, the combination of the nearest three nodes to $X_{R P}^{i}$ was shown at Eq. (4):

$$
\left\{\begin{array}{c|c}
\left\{X_{1}^{i}, X_{j}^{i}, X_{k}^{i}\right\} & \begin{array}{c}
X_{1}^{i} \in A^{i} ; X_{j}^{i} \in A^{i} ; X_{k}^{i} \in A^{i} ; \\
j, k=1,2, . ., n ; j \neq k \neq 1
\end{array}
\end{array}\right\}
$$

The temporary estimated coordinate for $X_{1 j k}^{i}$ was expressed at Eq. (5).

$$
X_{1 j k}^{i}=\left(x_{1 j k}^{i}=\frac{x_{1}^{i}+x_{j}^{i}+x_{k}^{i}}{3}, y_{1 j k}^{i}=\frac{y_{1}^{i}+y_{j}^{i}+y_{k}^{i}}{3}\right)
$$

In the same way, the temporary estimated coordinate for $X_{2 j k}^{i}, \ldots X_{N j k}^{i}$ can be obtained. The distance of each temporary coordinates with the reference nodes can be calculated as Eq. (6).

$$
d_{x_{m j k}^{i}}^{i}=\left\|X_{m j k}^{i}-X_{R P}^{i}\right\|, m, j, k=1,2, . ., N ; j \neq k \neq m
$$

For each combination set, found the minimum value of distance, obtained the temporary estimation coordinate as an average coordinate with the shortest distance of each set, as expressed at Eq. (7). 


$$
\left\{\begin{array}{c}
X_{t 1}^{i}=\left\{X_{t e 1}^{i} \mid \begin{array}{c}
\min \left\{d_{x_{1 j k}^{i j}}\right\} ; X_{t e 1}^{i} \in\left\{X_{1 j k}^{i}\right\} ; \\
j, k=1,2, \ldots, n ; j \neq k \neq 1
\end{array}\right\} \\
X_{t 2}^{i}=\left\{\begin{array}{c}
X_{t e 2}^{i} \mid \begin{array}{c}
\min \left\{d_{x_{2 j k}^{i}}\right\} ; X_{t e 2}^{i} \in\left\{X_{2 j k}^{i}\right\} \\
j, k=1,2, \ldots, n ; j \neq k \neq 1
\end{array} \\
\ldots
\end{array}\right\} \\
X_{t n}^{i}=\left\{\begin{array}{l}
X_{t e n}^{i} \mid \begin{array}{l}
\min \left\{d_{x_{n j k}^{i}}\right\} \\
j, k=1,2, \ldots, n ; j \neq k \neq 1
\end{array}
\end{array}\right\} X_{t e n}^{i} \in\left\{X_{n j k}^{i}\right\} ;
\end{array}\right.
$$

The final estimated coordinate of unknown node, $i$, became (8).

$$
X_{e s t}^{i}=\left(x_{e s t}^{i}=\frac{x_{t 1}^{i}+x_{t 2}^{i}+\ldots+x_{t N}^{i}}{N}, y_{e s t}^{i}=\frac{y_{t 1}^{i}+y_{t 2}^{i}+\ldots+y_{t N}^{i}}{N}\right)
$$

\subsection{Recursive Position Estimation Algorithm for Wireless Sensor Networks}

Recursive position estimation algorithm has very helpful in the condition of low density sensor networks. The basic concept of this algorithm is described as follows: at first, it calculates the position of unknown nodes using specific calculation method. The estimated node becomes to be the settled node. This settled node together with the previous reference node can be selected to be pairs of the remaining nodes on calculating their position. The process is employed repeatedly. If all unknown nodes positions have been estimated then the process will be finished.

Direct Position Algorithm proposed by de Oliveira, et al. [5] explained that the unknown nodes were estimated directly from its reference. While the settled node was generated it will become as a reference for the other unknown nodes. The process was performed repeatedly. The algorithm includes four steps:

Step 1. Beginning the Recursion Process from a Single Node

In this step, select four references with a given distance from a central point (the origin of recursion process), and the angle between each pair of neighbor beacons is known. Then, the base of the relative coordinate system is formed by the positions of the recursion origin $(0,0)$, the first beacon node $(5,0)$, and the second beacon node $(0,5)$. To start the recursion, the beacon nodes send a packet containing their position information. 
Step 2. Select the set of reference sensors

The selection process of reference nodes is decided by declares which references and settled node allowed to be the pairs of the remaining unknown nodes.

Step 3. Calculate the estimated position

This process has been done while the destination nodes were still unknown nodes. The position of unknown nodes can be calculated with several kinds of methods as explained before. In this step, we can add the optimization models to reduce the error estimated position.

Step 4. Send the information packet

The unknown node with estimated position may declare its status as a settled node to the whole neighbour nodes by sending its position in a kind of packet. The other unknown nodes may employ this settled node as its reference to estimates their position.

\section{$4 \quad$ The Proposed Centroid Algorithms}

The previous research by Kristalina, et al. [11], had described the proposed IWC Algorithm. IWC Algorithm or Improved Weighted Connectivity based Algorithm, was an effort to improve the fundamental centroid algorithm with consideration of connectivity and distance of involved reference nodes to an unknown nodes, respectively. It assumed that the closer the position of reference nodes from their neighbour unknown nodes the bigger the accuracy estimated position could be reached by those unknown nodes using Centroid Algorithm. The effect of inter node connectivity, as depicted at Figure 3.

There are two types of the proposed IWC Centroid algorithm: Connectivitybased Centroid (CB Centroid) and Improved Connectivity-based Centroid (ICB). The weighting is given with the assumptions as follows: each unknown node has availability to receive the signal from reference node with the same sensitivity, or in the other hand, the same communication range is implemented to each unknown node. Moreover, each unknown node can be set to be able to receive the signal from any direction.

\subsection{Connectivity-based Centroid Algorithm}

The availability of unknown nodes in their communication range has to be considered to reach a number of neighbor reference nodes. It can be stated that each reference sensor has weighted factor to the involved unknown nodes. The weighted factor is inversely proportional with the distance measurement of 
reference and the respective unknown node. Generally, the closer the reference position to the unknown node the bigger the weighted factor is. Figure 4 shows the related of connectivity weight and the distance between two nodes.
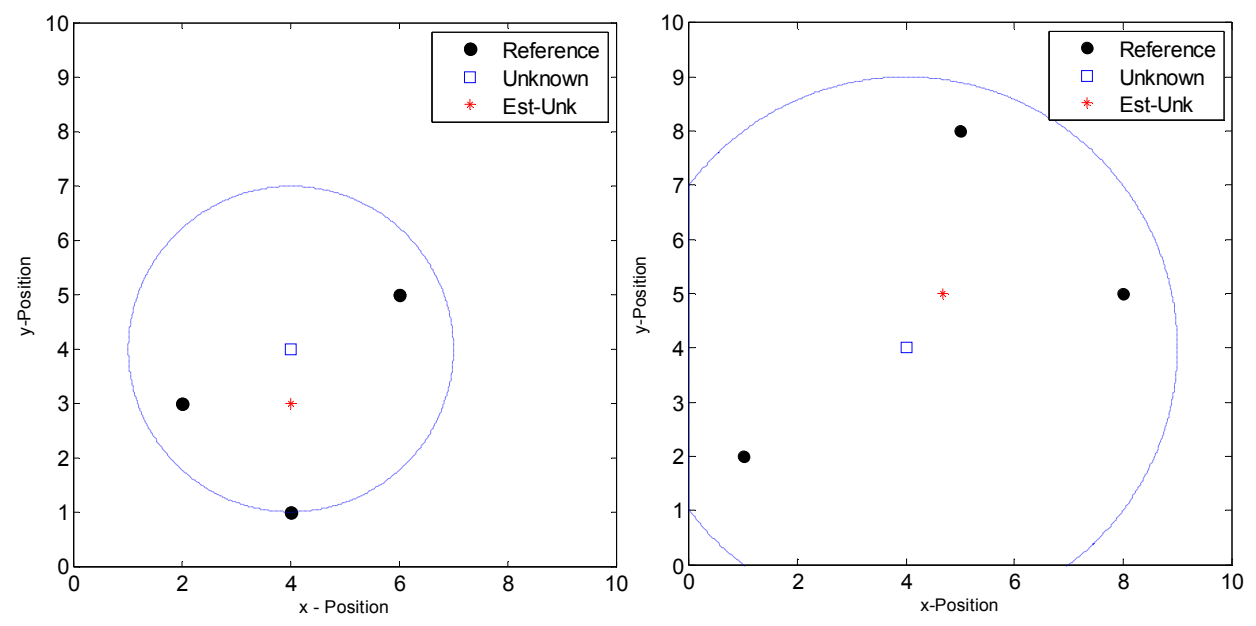

Figure 3 The effect of inter node connectivity on Estimation Node Position Accuracy

For all $i \in B$ and $j \in U$ where $\{B, U\} \in N$ thus the weighted factor is expressed at Eq. (9).

$$
w_{i j}=\frac{1}{d_{i j}}
$$

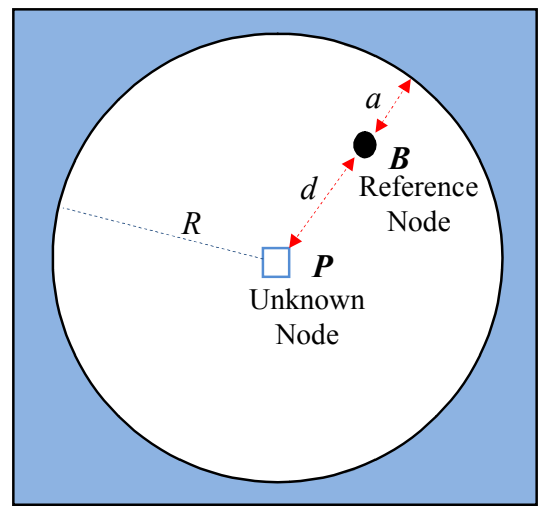

Figure 4 The connectivity weight and the distance between two nodes 
The estimated position for unknown node, $i$, with the communication range $R$, and distance $d$ from any reference nodes inside the unknown communication range is expressed at Eq. (10).

$$
\left(X_{e s t}^{i}, Y_{e s t}^{i}\right)=\left(\frac{\sum_{j=1}^{N_{B}} w_{i j} x_{i j}}{N_{B}}, \frac{\sum_{j=1}^{N_{B}} w_{i j} y_{i j}}{N_{B}}\right)
$$

Where $N_{B}$ is the number of reference nodes inside the unknown communication range, R. Connectivity-based Centroid Algorithm proposed is explained at Algorithm 1.

Algorithm 1.

Initialization:

1. Define Area $=[0$ BorderLength 0 BorderLength $]$

2. Input : Reference Nodes Position $\left(X_{j}, Y_{j}\right), j \in B$

3. Unknown Nodes Number, $N$

4. Communication Range: $0.5 B L<R<=B L$

FOR $i=1: N$

$\operatorname{FOR} j=1: B$ (if this node is Reference)

Distance Measurement:

5. Input $d_{i j}$ (measured distance of unknown $i$ from reference $j$ )

Calculate Estimated Position:

6. IF $d_{i j}<R$

7. FOR $k=1: N_{B} \quad$ (number of reference enclosed by unknown node communication range $R$ )

8. $\quad w_{i k}=1 / d_{i k}$

9. $\left(X_{\text {est }}^{i}, Y_{\text {est }}^{i}\right)=\left(\frac{\sum_{k=1}^{N_{B}} w_{i k} x_{i k}}{N_{B}}, \frac{\sum_{k=1}^{N_{B}} w_{i k} y_{i k}}{N_{B}}\right)$

10. END IF

Error Measurement:

FOR $i=1: N$

11. $E r r_{i}=\sqrt{\left(X_{a c t}^{i}-X_{e s t}^{i}\right)^{2}+\left(Y_{a c t}^{i}-Y_{e s t}^{i}\right)^{2}}$ 


\subsection{Improved Connectivity-based Centroid Algorithm}

This algorithm is the extension phase of the previous one, i.e Connectivitybased Centroid Algorithm. The area of communication range is divided into several parts. This statement is based in the fact that in the future time, sensors have the availability on self-manage dynamically. Through this availability, the sensors could reach the references in the vicinity. According to the power control mechanism, an unknown node can applies self control where it needs more reference nodes to estimate its position. Figure 5 shows the division of communication area of an unknown node. The farther the area from central point is the lower the connection weight possessed. Each node rest in the same area has the same value of connection weight. Improved Connectivity-based Centroid Algorithm proposed is given as Algorithm 2.

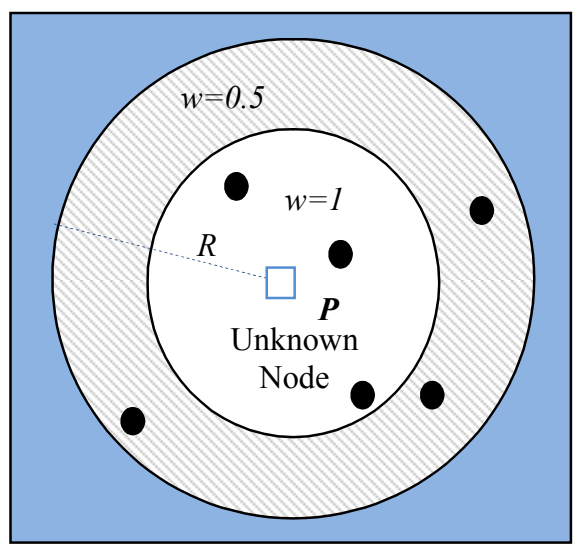

Figure 5 The weighted Division Area.

\subsection{Recursive Position Estimation to improve Connection-based Centroid Algorithm}

The Centroid proposed algorithm calculates the position of an unknown node. The shortcoming factor of this algorithm is the dependency of each unknown nodes to the existing reference nodes. Non distributed reference nodes topology might be found and cause reduction on accuracy. We propose Recursive Position Estimation, the localization method on finding the estimated position of unknown nodes by recursion process. The process starts from any unknown node with three reference nodes. This node is estimated using Basic Centroid. When the result occurs, this become a settled node, the node together with existing reference nodes will become as reference nodes for the remain 
unknown nodes. The process repeats until all unknown nodes has been estimating their position. The algorithm of RPE is illustrated as Algorithm 3.

Algorithm 2.

1-5 similar with Algoritma 1.

Calculate Estimated Position:

6. IF $d_{i j}<R$

7. IF $d_{i j}<=0.5 R$

8. FOR $k=1: N_{B 1} \quad$ (number of reference enclosed by unknown communication range $0.5 R$ )

9. $w_{i k}=1$

\section{ELSE IF}

10. FOR $k=1: N_{B 2} \quad$ (number of reference enclosed by unknown communication range $0.5 R<d_{i j}<R$ )

11. $w_{i k}=0.5$

12. $\left(X_{\text {est }}^{i}, Y_{e s t}^{i}\right)=\left(\frac{\sum_{k=1}^{N_{B}} w_{i k} x_{i k}}{N_{B}}, \frac{\sum_{k=1}^{N_{B}} w_{i k} y_{i k}}{N_{B}}\right)$

\section{END IF}

\section{Error Measurement:}

FOR $i=1: N$

14. $\operatorname{Err}_{i}=\sqrt{\left(X_{a c t}^{i}-X_{e s t}^{i}\right)^{2}+\left(Y_{a c t}^{i}-Y_{e s t}^{i}\right)^{2}}$

Algorithm 3.

1. Initialization

2. Round $=0$

3. SettleNode $=0$

4. While Round $<$ MaxRound

5. Round $=$ Round +1

6. IF this node is a Reference Node THEN

7. Send its location data to neighboring node

8. ELSE IF this node is NOT Reference Node \&\& UnknownNo $>$ minUnk

9. Get the 3 minimum Distance from its previous anchor

10. Calculate its position using IWB Centroid

11. Select as a SettleNode

12. Update Anchor List

13. Update Unknown List

14. ELSEIF UnknownNode $=$ minUnk

15. Stop Localization

16. ENDIF 


\section{Simulation Results and Discussion}

Simulation was done on Matlab ver.7.8 running at 1,4 GHz PC with 4 GB memory. The simulation parameters are listed at Table 1 .

The observations were includes: the Average Error Position obtained from increasing the number of nodes, communication range, area and simulation time required for each estimating process. These observations were conducted on $\mathrm{CB}$ Centroid Algorithm and ICB Centroid Algorithm, whereas Recursive Position Estimation was observed to obtain the average error position against the settled node numbers, the computation time and the increasing of error reduction.

Table 1 Table of Simulation Parameters.

\begin{tabular}{llll}
\hline Variable & Definition & Range & Meas.Unit \\
\hline Grid & Grid Size & $3-10$ & Pcs \\
NA & $\begin{array}{l}\text { Number of } \\
\text { Reference node }\end{array}$ & Grid $^{2}$ & Pcs \\
NU & $\begin{array}{l}\text { Number of Unknown } \\
\text { node }\end{array}$ & $4-80$ & Pcs \\
& Border Length & $2-100$ & meters \\
R & $\begin{array}{l}\text { Communication } \\
\text { range }\end{array}$ & $0.5^{*} \mathrm{BL}-\mathrm{BL}$ & meters \\
Err & $\begin{array}{l}\text { Error of Position } \\
\text { Estimation }\end{array}$ & $0-10$ & meters \\
iter & Iteration & 100 & times \\
\hline
\end{tabular}

Nodes were deployed inside two dimension area $\left[\begin{array}{llll}0 & \mathrm{BL} & 0 & \mathrm{BL}\end{array}\right]$ using grid topology for reference nodes and random for unknown nodes, as shown at Figure 6 , followed by calculating the position using the proposed algorithm. The simulation results were compared with the fundamental Centroid, as depicted at Figure 7.

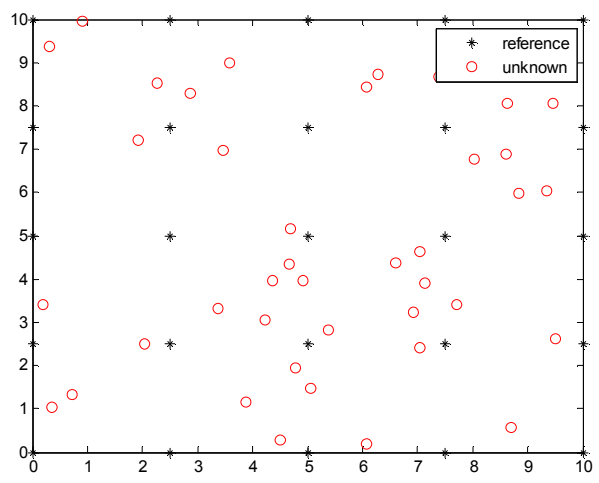

Figure 6 Node Deployment (Grid for References and Random for Unknown). 


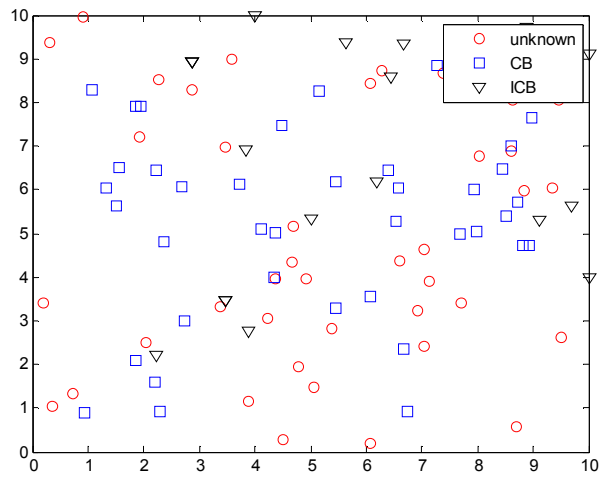

Figure 7 Simulation result for estimated unknown using $\mathrm{CB}$ and ICB algorithms after 20 times iteration.

\subsection{Number of Reference Nodes vs Average Error Position}

In the experiment with the fixed communication range and number of unknown nodes, we deploy varying number of reference nodes. The result as shown in Figure 8, explained that enhancing the nodes cause an increase of average error position. The increasing trend will be saturated when the number of reference nodes exceeds the number of existing unknown. This result is apply both for grid or random deployment, in small or wider area. Based on our analyze, in the area with certain number of unknown nodes, where each unknown node has the same communication range, the more nodes deployed the more chances to be a reference for the unknown node involved. Unfortunately, however, the overlap between two or more unknown nodes will increase. This phenomena shown why the error position is increased followed by saturated condition. The best performance may obtained when the ratio of reference and unknown nodes is $10 \%$.

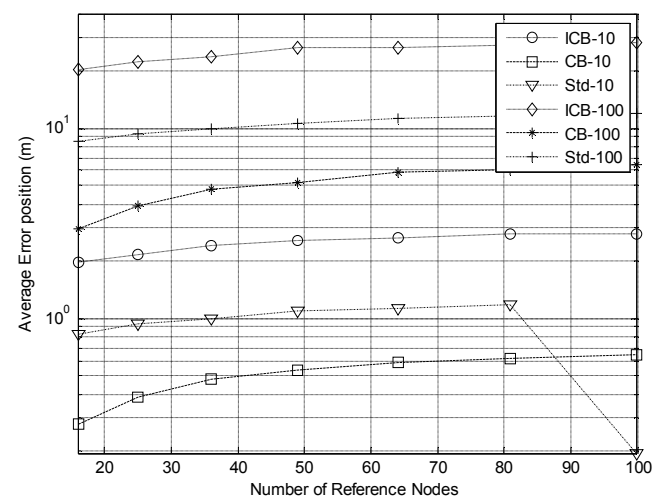

Figure 8 Number of reference nodes vs Average Error Position for $\mathrm{NU}=50$, $\mathrm{BL}=10-100, \mathrm{R}=5-50$. 


\subsection{Communication Range vs Average Error Position}

The communication range of the unknown node related to the capability of the node to make the communication with the reference nodes around it. Figure 9 shows the effect of adding the communication range of each unknown node to the average error position. The curve shows that the average error position increases as the communication range is enlarged. We do the analysis that, the greater the radius of a circle the more nodes are involved. While the position of reference nodes are far away from the unknown node, then the accuracy of estimation position becomes lower.

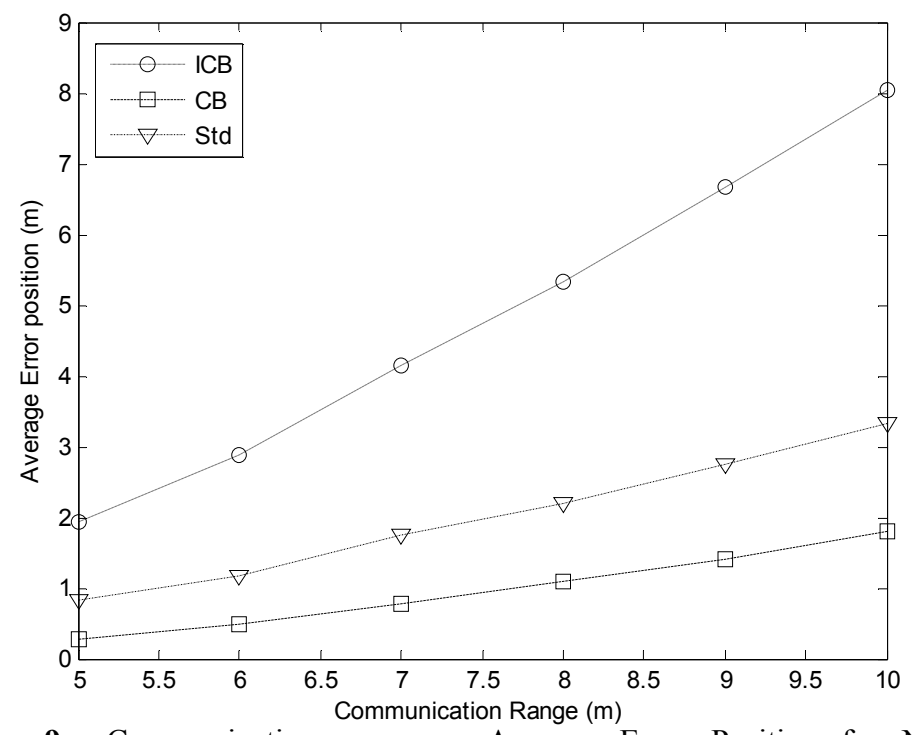

Figure 9 Communication range vs Average Error Position for $\mathrm{NA}=16$, $\mathrm{NU}=50, \mathrm{BL}=10$.

\subsection{Number of Unknown Nodes vs Average Error Position}

The unknown nodes were randomly deployed in the fixed area (10x10) where 3 reference nodes with fix position were existed. The number of these nodes was increased from 3 to 30 . Figure 10 shows that the average error position is increased as the number of unknown nodes increase. We can describe that as more unknown nodes might impose their estimation process the distance between them and their reference might be more varied. Thus, the average error position will be greater. The best performance is shown by $\mathrm{CB}$ Centroid algorithm, with $66,67 \%$ better accuracy than Basic Centroid. The worst one is performed by ICB Centroid. 


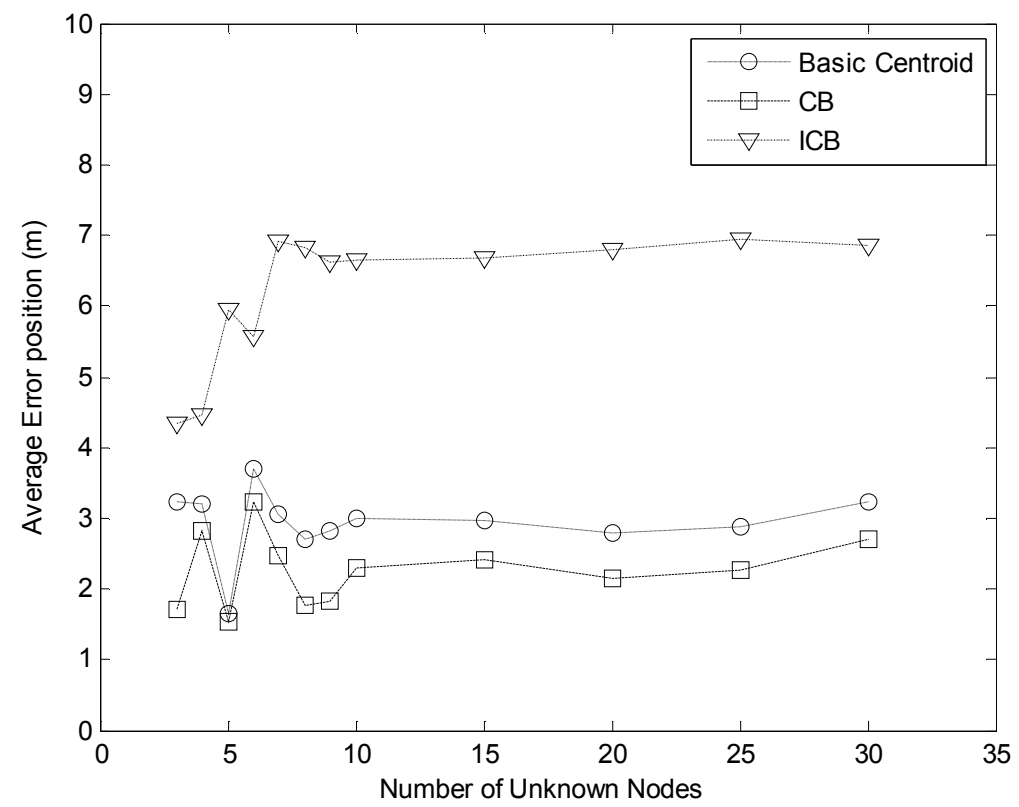

Figure 10 Number of Unknown Nodes vs Average Error Position for NA=3, $\mathrm{NU}=3-30, \mathrm{BL}=10$.

\subsection{Time of Simulation vs Average Error Position}

The average error position for $\mathrm{NA}=25, \mathrm{NU}=50, \mathrm{BL}=10, \mathrm{R}=5$ is observed 50 times of iteration. In ICB Centroid Algorithm, the minimum error obtained is $2.4337 \mathrm{~m}$, and the maximum error is $3.3657 \mathrm{~m}$, as illustrated at Figure 11 . The deviation error is $0.932 \mathrm{~m}$. In CB Centroid algorithm, the minimum error obtained is $0.6135 \mathrm{~m}$, and the maximum error is $0.9046 \mathrm{~m}$, as shown at Fig. 12 . The deviation error is $0.2911 \mathrm{~m}$. Fundamental Centroid has minimum error $1.0101 \mathrm{~m}$ and maximum error $1.4264 \mathrm{~m}$, and deviation error $0.4163 \mathrm{~m}$.

The explanation shows that the accuracy level of ICB algorithm is 4 times worse than $\mathrm{CB}$ algorithm, or in the other words, performance of $\mathrm{CB}$ algorithm is 4 times better than ICB algorithm. Moreover, CB algorithm has capability to improve error position of unknown nodes 1.65 times better than the fundamental Centroid. 


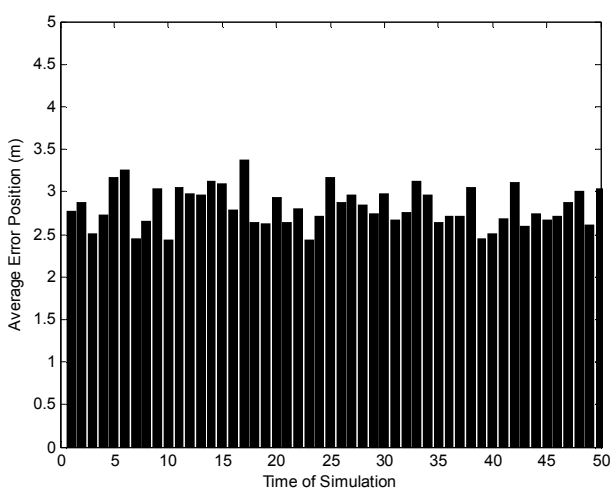

(a)

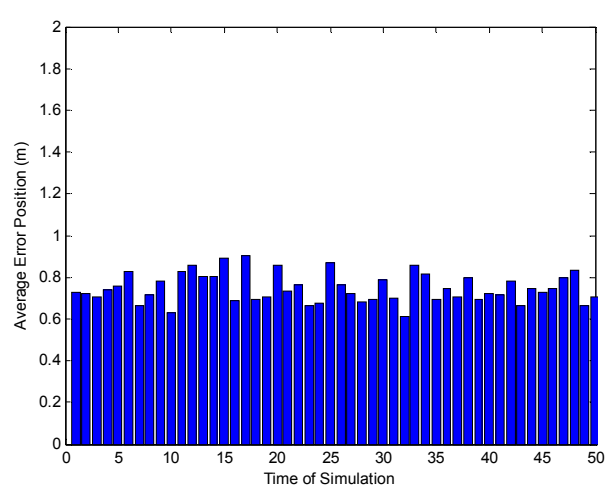

(b)

Figure 11 a. CB Centroid; b. ICB Centroid.

\subsection{RPE Improvement vs Average Error Position}

The previous observation shows that ICB Centroid has failed in improving the performance of Centroid. We propose Recursive Position Estimation Algorithm (RPE Algorithm) instead of ICB. Figure 12 shows that RPE for Basic Centroid enables to increase the performance up to $50 \%$. The error residue due to settled nodes establishment causes the decreasing of RPE Algorithm. The decrement performance is up to twice worse than Basic Centroid Algorithm.

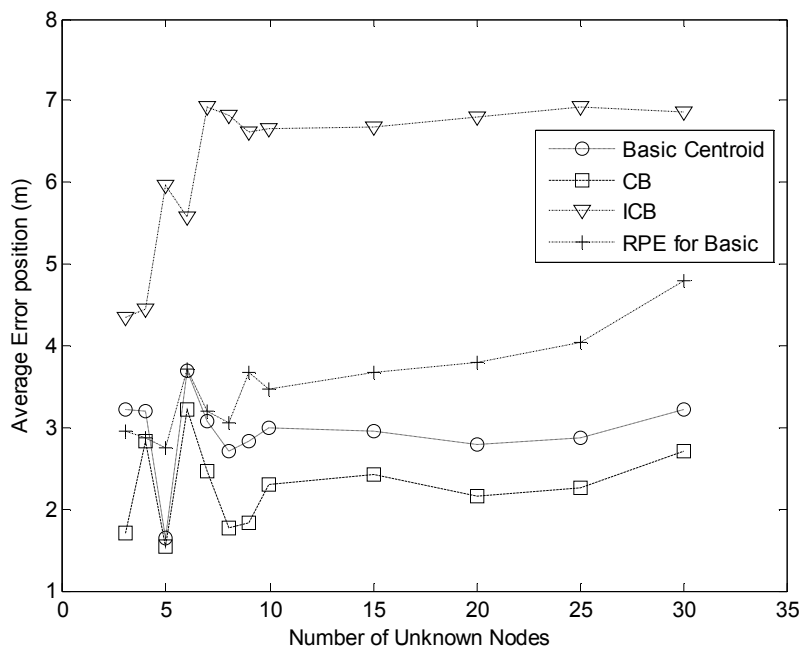

Figure 12 RPE Improvement on Average Error Position. 


\subsection{Comparison the Average Computation Time of Proposed Algorithms}

Computation time indicates the time required on finishing the estimation procedures. The basic Centroid and CB Centroid Algorithm are imposed simultaneously in each deployed node so that the computation time are faster than the RPE Algorithm where the process is taken more time in the aim of sequentially process of settled node generation. As we described in the last section that settled node is generated from the previous centroid process. Moreover, the set of nodes includes the settled nodes and reference nodes are employed to calculate the estimation position of the remaining nodes. The process will be done repeatedly until the position of whole nodes is well estimated. The comparison of average time calculation of three proposed algorithms is shown at Figure 13.

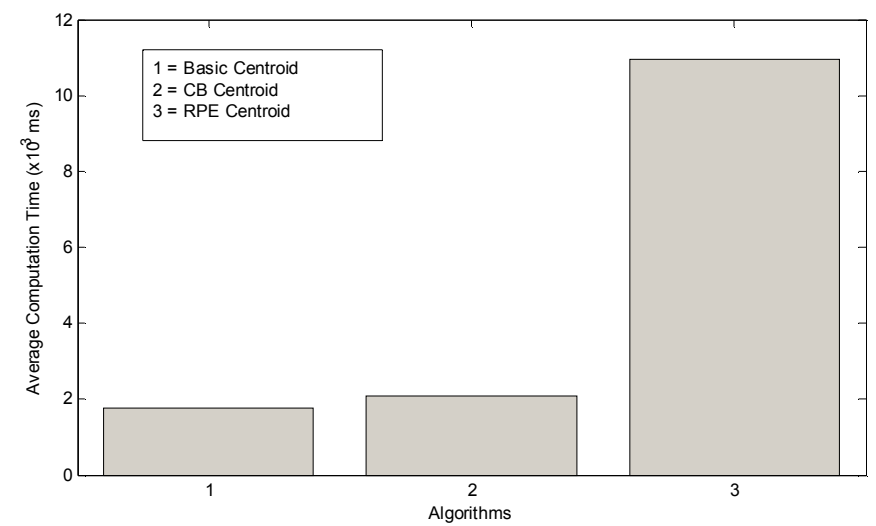

Figure 13 The comparison of average time calculation of three proposed algorithms.

\section{Conclusions}

Knowing the connectivity between unknown nodes to the neighbor reference nodes is very essential to increase the accuracy of estimated position. The proposed IWC Centroid Algorithm results the better performance on minimize the average error position than basic Centroid, yet the ICB Algorithm performs slightly poor accuracy. Recursive Position Estimation algorithm is exploited in order to reduce the average error position. The simulation results that the RPE Algorithm has the ability on improving the performance of ICB Algorithm up to $50 \%$. The required calculation time of RPE is due to the sequentially process of settled node generation. Considering the connectivity, it could be realized to design the sensor node which has capability to control its communication range dynamically in order to get the maximum accuracy on wireless sensor networks. 


\section{References}

[1] Liu, Y. \& Yang, Z., Location, Localization, and Localizability: Locationawareness Technology for Wireless Networks, $1^{\text {st }}$ ed., New York, USA: Springer, 2011.

[2] Deng, B., Huang, G., Zhang, L. \& Liu, H., Improved Centroid Localization Algorithm in WSNs, Proc. $3^{\text {rd }}$ Int' 1 Conf. on Intelligent System and Knowledge Engineering (ICISKE), pp.1260-1264, 2008.

[3] Cheng, H., Wang, H. \& Wang, H., Research on Centroid Localization Algorithm that Uses Modified Weight in WSN, Proc. 2011 Int'l Conf. on Network Computing and Information Security (ICNCIS), pp.287-291, 2011.

[4] Boukerche, A., Algorithms and Protocols for Wireless Sensor Networks, $1^{\text {st }}$ ed., New Jersey, USA: John Wiley \& Sons, 2009.

[5] de Olivera, H.A.B.F., Boukerche, A., Nakamura, E.F., Loureiro, A.A.F., An Efficient Directed Localization Recursion Protocol for Wireless Sensor Networks, IEEE Trans on Comp., 58(5), pp.677 - 691, 2009.

[6] Mao, G. \& Fidan, B., Localization Algorithms and Strategies for Wireless Sensor Networks, $1^{\text {st }}$ ed., Hershey, USA: Information Science Reference, 2009.

[7] Langendoen, K. \& Reijers, N., Distributed Localization in Wireless Sensor Networks: A Quantitative Comparison, Journal on Computer Networks, 43, pp. 499-518, 2003.

[8] Shi, Q., Huo, H., Fang, T. \& Li, D., A Distributed Node Localization Scheme for Wireless Sensor Networks, Wireless Personal Communication Journal, 53, pp. 15-33, 2010.

[9] Bulusu, N., Heidemann, J. \& Estrin, D., GPS-Less Low Cost Outdoor Localization for Very Small Devices, IEEE Pers. Commun., 7(5), pp. 2834, 2000.

[10] Yun, S., Lee, J., Chung, W. \& Kim, E., Centroid Localization Method in Wireless Sensor Networks using TSK Fuzzy Modelling, Proc. $6^{\text {th }}$ Int'1 Symps. On Advanced Intelligent System (ISIS), pp. 971-974, 2005.

[11] Kristalina, P., Wirawan \& Hendrantoro, G., Improved Range-free Localization Methods for Wireless Sensor Networks, Proc. 2011 Int'1 Conf. on Electrical Engineering and Informatic (ICEEI), pp. I5-1, 2011. 